\title{
Effect of Different ECG Leads on Estimated R-R Intervals and Heart Rate Variability Parameters
}

\author{
Vala Jeyhani $^{1}$, Matti Mäntysalo ${ }^{2}$, Kai Noponen ${ }^{3}$, Tapio Seppänen ${ }^{3}$ and Antti Vehkaoja ${ }^{1}$
}

\begin{abstract}
Heart rate and heart rate variability parameters provide important information on sympathetic and parasympathetic branches of autonomous nervous system. These parameters are usually extracted from electrocardiograms often measured between two electrodes and called an ECG lead. Besides systems intended only for heart rate measurement, ECG measurement devices employ several well-known lead systems including the standard 12-lead system, EASI lead system and Mason-Likar systems. Therefore, the first step is to select the appropriate lead for heart rate variability analysis. The appropriate electrode locations for single-lead measurement systems or the preferred measurement lead in multi-lead measurement are choices that the user needs to make when the heart rate variability is of interest. However, it has not been addressed in the literature, if the lead selection has an effect on the obtained HRV parameters. In this work, we characterized the amount of deviation of heart rate and heart rate variability parameters extracted from nine ECG leads, six from EASI leads and three modified limb leads. The results showed a deviation of $2.04,2.88,2.06$ and 3.45 ms in SDNN, rMSSD, SD1 and SD2, respectively. A relative difference up to $10 \%$ was observed in HRV parameters for single signal frames. Additionally, the discrimination of the Rpeaks by amplitudes was evaluated. The A-S lead appeared to have the best performance in all the tests.
\end{abstract}

\section{INTRODUCTION}

Heart rate (HR) is mainly regulated by activity of the sympathetic and parasympathetic branches of autonomous nervous system (ANS) and heart rate variability (HRV) is a popular marker used for assessing the status of ANS. HR and HRV have for example been utilized in characterizing the fitness level [1] [2] and in assessing stress [3]. Furthermore, it is also shown that HRV is correlated with responses in the tasks associated with memory and attention [4].

Electrocardiography (ECG) is the most common method used in heart rate and HRV monitoring while photoplethysmography (PPG) and ballistocardiography are increasingly

*This research was funded by the Finnish Funding Agency for Innovation (Tekes) and several Finnish companies as a part of VitalSens project. Funding decision number: 40103/14. Matti Mäntysalo and Antti Vehkaoja are supported by Academy of Finland (grant no. 288945 and 294119 for MM and grant no. 292477 for AV)

${ }^{1}$ Vala Jeyhani and Antti Vehkaoja are with BioMediTech Institute and Faculty of Medicine and Health Technology, Tampere University, Tampere, Finland valajeyhani@gmail.com antti.vehkaoja@tuni.fi

${ }^{2}$ Matti Mäntysalo is with the Faculty of Information Technology and Communication Sciences, Tampere University, Tampere, Finland matti.mantysalo@tuni.fi

${ }^{3}$ Kai Noponen and Tapio Seppänen are with Physiological Signal Analysis Group, Center for Machine Vision and Signal Analysis, University of Oulu, Oulu, Finland kai.noponen@oulu.fi tapio.seppanen@oulu.fi popular alternatives for data collection due to their unobtrusiveness [5] [6]. HR and HRV analysis is performed either offline, after the recording is done, usually by experts assisted by computer tools, or in real-time, which may also be associated with wireless data transmission and online monitoring of these parameters. Wearable devices and fitness trackers have become powerful enough to perform this type of analysis. From power and memory consumption point of view, on-line analysis and transmission of only the HR and HRV information may be the most efficient approach even though the ECG sampling frequency as low as $50 \mathrm{~Hz}$ has been shown adequate for HRV analysis [7].

Some of these devices provide the possibility of measuring several ECG leads and some others, which monitor and record only HR information, may be attached to different locations on the chest area. In either cases, the question is, which lead or electrode location provides the most robust result and further whether the electrode location or the measurement lead has an effect on the HRV parameters resulted from them. The movements in thoracic area resulting from respiration and other sources influence the orientation of the heart with respect to the measurement lead and also the volume conductor formed by the body. While the measurement lead affects the exact temporal location of the R-peaks as illustrated in Fig. 1, the changes in the measurement geometry between R-peaks may have an effect on the R-R intervals and thus the HRV parameters.

There is limited research related to above questions, and the dominance of the QRS complex has been considered as the only factor for selecting the appropriate ECG lead. Puurtinen et al. studied the signal strength of the measured ECG from 120 unipolar leads [8]. They found the highest QRScomplex amplitude in the precordial ECG leads V2, V3 and V4 (with respect to the Wilson Terminal). They also showed that in all the leads the QRS-complexes were clearly higher in amplitude than the noise of $15 \mathrm{uV}$, which corresponds to a low noise situation. Noh et al. studied several chest electrode locations in order to find a suitable lead for a two-electrode ECG measurement [9]. They considered V1, V2, V3, V4 and V5 electrode locations along with two additional location above V1 and V2, around manubrium. They found that when one electrode is placed at V1 and another somewhere close to V5, the measured QRS has the clearest appearance among other leads. However, they did not consider all the possible leads among these electrode locations.

In this work, the deviation in HRV analysis resulted from using different ECG leads is studied. Nine ECG leads from commonly used electrode locations have been measured and 




Fig. 1. QRS complexes of different ECG leads showing the differences in R-peak amplitude and temporal locations. The two lead sets EASI and standard leads were measured by two individual devices. As a result, the two groups of signals might not by fully synchronous.

the HRV analysis has been performed on each of them individually. We have also investigated the clarity of QRS complexes among these different leads.

\section{MEASUREMENTS}

The measurements were performed using a custom developed device ${ }^{1}$ [10]. Three ECG leads (using four electrodes) from EASI lead set [11] (I-S, A-S and E-S) and two limb leads (leads I and II) from Mason-Likar [12] lead set were measured using two devices with a data rate of 1000 samples per second (sps). Fig. 2 shows the electrode locations. There was no analog high-pass filter in the measurement devices and the right-leg drive was not utilized. The measured signals were stored on the local memory of the device with a resolution of 24 bits. Conventional $\mathrm{Ag} / \mathrm{AgCl}$ electrodes were used for the measurements.

In total, five subjects who had no history of cardiac problems volunteered for the study. Informed consent was obtained from all the participants. Skin preparation, which included cleaning the skin with alcohol and removing the dead skin cells, was performed prior to the measurements. The electrode wires were attached to the body using a piece of tape to reduce movement artifacts in the measurements. The subjects were instructed to breathe normally and move as little as possible. The measurements were performed in supine position and lasted for 15 minutes for each subject.

\section{SIGNAL PROCESSING}

Additional leads were computed using the measured leads, resulting in the six leads of EASI lead set (i.e. I-S, A-S, ES, I-E, A-E and A-I) and three limb leads (I, II and III).

\footnotetext{
${ }^{1}$ A demonstration of the device can be found at www.spiritcor9d.xyz
}



Fig. 2. The electrode locations for measuring modified limb leads and EASI leads. Copyright: pushinka / 123RF Stock Photo

The lead A-E and the lead I were used for synchronizing the measurements from the two devices: First, the R-peaks were found from both of the selected leads. Next, the R-R interval (RRI) signal was calculated. Then, the RRI series resulted from lead I signal was shifted to right sample by sample and the absolute of the difference between the two RRI series was calculated. This process was repeated till a local minimum was found in the absolute of the differences. The index of this minima was considered as the needed amount of shift for all the limb leads. Finally, the synchronicity was confirmed visually.

\section{A. R-peak Detection}

For finding the R-peaks in the ECG signals, first, the baseline wandering was reduced by calculating the median of the signal in $100 \mathrm{~ms}$-long frames and subtracting these values from the original signal [13]. Next, the signals were filtered with a 3rd order low-pass filter having Bessel response and a cut-off frequency of $30 \mathrm{~Hz}$. This was done to minimize the effect of high frequency noise and artifacts on the Rpeak detection process. The filtering was performed using the forward-backward method to prevent the effect of the nonlinear phase response of the filter. Finally, the signals underwent an R-peak detection algorithm based on the method proposed by Zhengzhong et al. [14], which uses the history of the slopes and amplitudes of the R-peaks.

The process of R-peak detection is the following: The first 10 seconds of the signal was used as the initial learning process that was divided to five equal non-overlapping frames. Next, in each frame, the differences between consecutive samples were calculated and the maximum and minimum points, which correspond to the fastest ascending and descending slopes in the ECG signal, were found. Then, a range was defined between these two points and the maximum value of the original signal in this range was located. These three values from the five frames acted as the basis of the thresholds for the future segments of the signal. The multipliers for these thresholds were set to 0.15 , 0.15 and 0.55 for the maximum slope, minimum slope and local maximum of the signal, respectively. The rest of the signal was examined against the thresholds in the form of 10 second frames. The frames were moved with a 1 second step and each frame was windowed using a Hanning window 

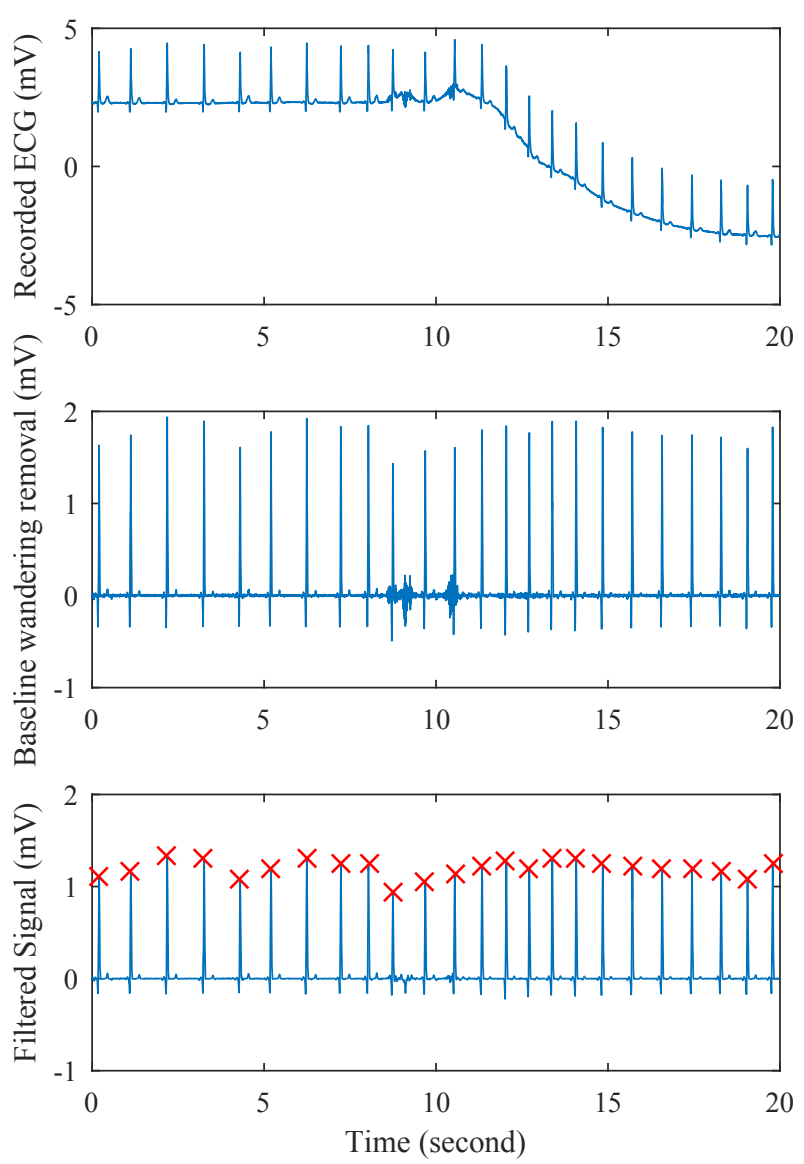

Fig. 3. The recorded ECG signal (top), the result of baseline wandering removal (middle) and the output of the filtering and R-peak detection algorithm (bottom).

to prevent possible edge effects. The found R-peaks in each frame was compared to the list of previously found peaks to prevent storing an R-peak for multiple-times. The found and confirmed R-peaks were used to update the thresholds.

The result of baseline wandering correction and R-peak detection is illustrated in Fig. 3. Finally, all the RRI series from all the leads were compared and visually investigated. There were equal amount of samples for each lead and each subject.

\section{B. HRV Parameters}

Four HRV parameters were evaluated in this study, namely SDNN, rMSSD, SD1 and SD2. The definitions of these parameters are as follows [15]:

- SDNN: standard deviation of RRI

- rMSSD: root mean-square of successive differences of adjacent $\mathrm{R}-\mathrm{R}$ intervals

- SD1: standard deviation of data against the axis $\mathrm{x}=\mathrm{y}$ in Poincaré plot

- SD2: standard deviation of data against the axis, which is orthogonal to the axis $\mathrm{x}=\mathrm{y}$ and crosses this axis at the mean value of the data in Poincareé plot.

The HRV parameters were calculated for frames of RRI series that had a length of five minutes and were shifted by
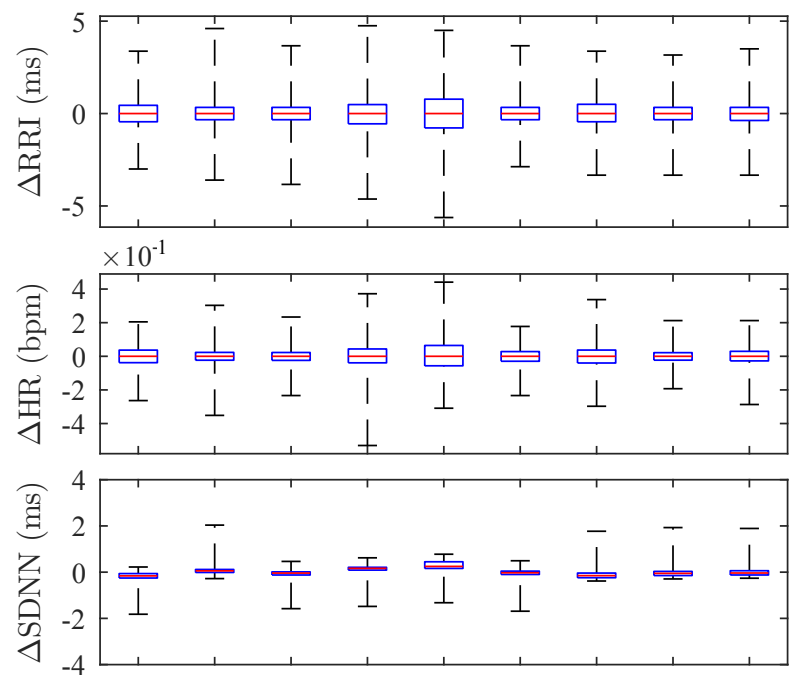


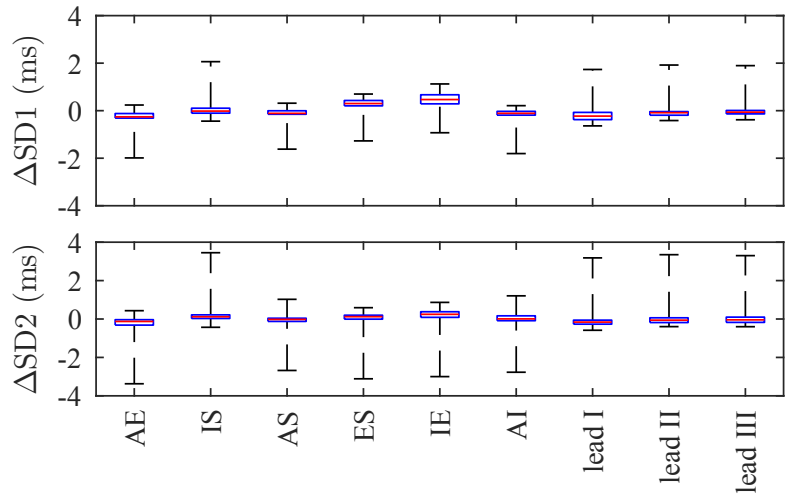

Fig. 4. The difference between the RRI and its corresponding mean calculated between all the leads (top). The same difference is illustrated for heart rate, SDNN, rMSSD, SD1 and SD2. The red lines in the boxes represent the median value. The top and bottom edges of the boxes indicated the 25th and 75th percentiles. The whiskers are extended to the maximum and minimum value.

thirty seconds, thus producing 30 frames for each lead. The frames were created based on the indices of the RRI series, which were inherited from their corresponding R-peaks.

\section{RESULTS}

The amount of interference was extremely small in all the measurements and leads. Fig. 3 shows one of the most corrupted parts of the signals, which was a result of coughing. The topmost panel shows a part of the signal measured from lead A-E. The result of the baseline wandering correction is depicted in the middle panel. The bottom panel shows the effect of the low pass-filtering. The found R-peaks are marked by red crosses. 

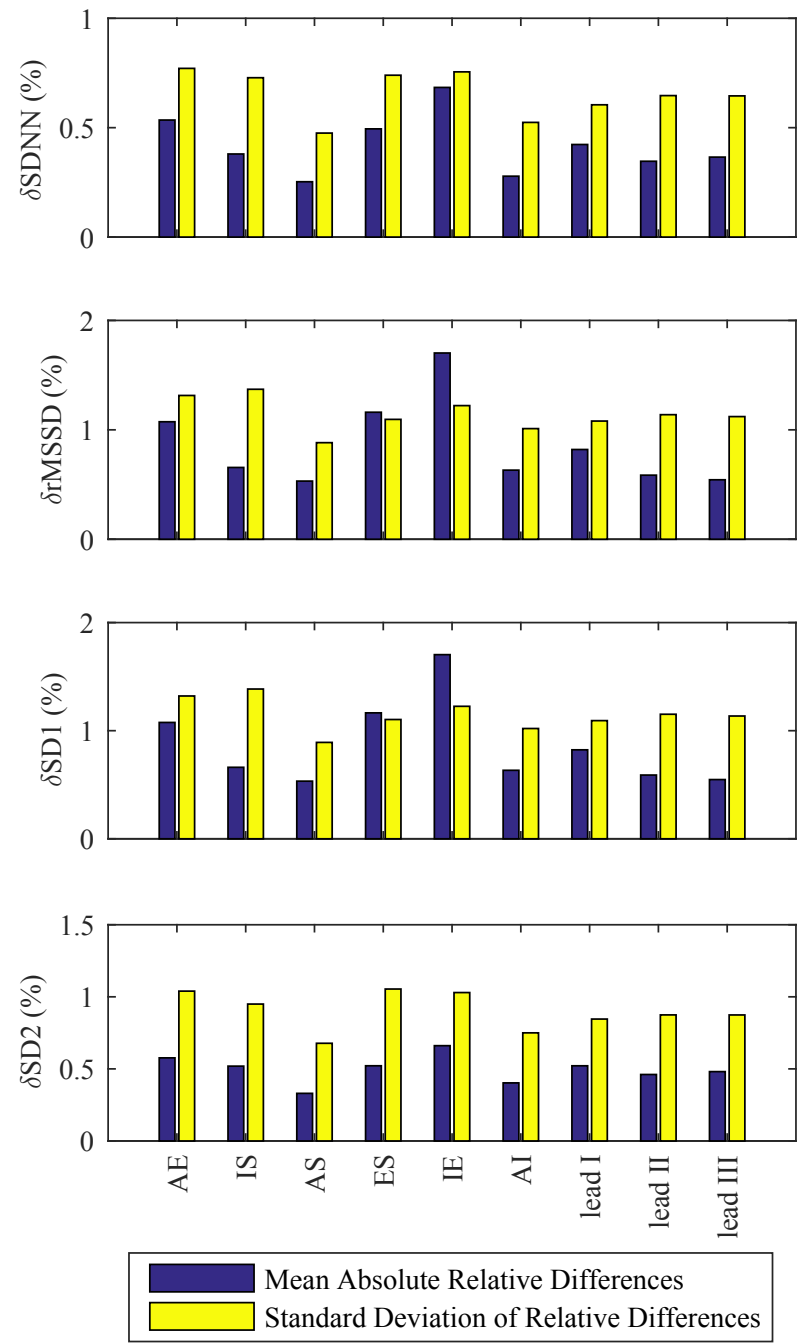

Fig. 5. The mean absolute and standard deviation of the relative differences

\section{A. RRI and HRV Parameters}

To evaluate the effect of electrode locations on HR, HRV and RRI parameters, the difference between each individual value and the average value of that parameter resulted from all the leads was calculated. This process was performed for each temporal frame and all the parameters. The results for RRI and HR are depicted in the first two panels of Fig. 4. The I-A lead was removed for one of the subjects from all the calculations due to a weak amplitude and therefore unreliable R-peak detection results. It can be seen that the maximum absolute deviation in the obtained $\mathrm{R}-\mathrm{R}$ intervals has been approximate $5 \mathrm{~ms}$ from the average, which happens in the I-E lead. When converted to heart rate, this corresponds roughly $0.4 \mathrm{bpm}$. Deviating from the HRV parameters, the results for the heart rate and the RRI are calculated using instantaneous values rather than the average HR or RRI within a frame.

The four bottom panels of Fig. 4 show the deviation from the mean for the four HRV parameters. The maximum deviations for SDNN, rMSSD, SD1 and SD2 were 2.04,

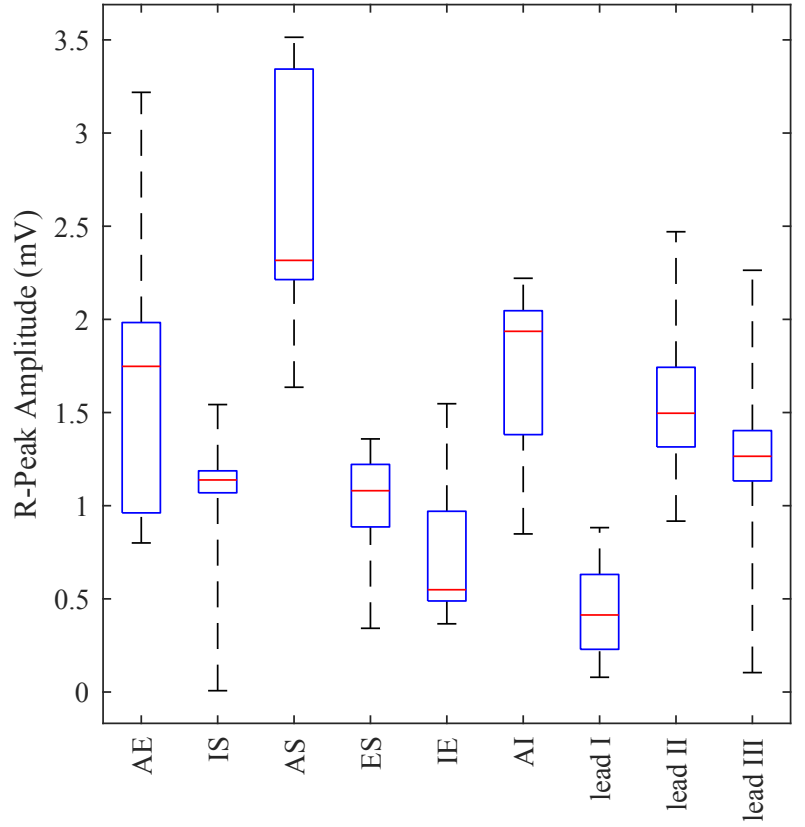

Fig. 6. R-peak amplitudes for each in all the subjects. The red lines in the boxes represent the median value. The top and bottom edges of the boxes indicated the 25 th and 75 th percentiles. The whiskers are extended to the maximum and minimum value.

$2.88,2.06$ and $3.45 \mathrm{~ms}$, respectively. It is worth noting that compared to the mean values, these deviations appear as overestimation for the I-S and the three limb leads and underestimation in the rest of the EASI leads.

In Fig. 5, the deviations are presented as the relative difference percentages, which are calculated by dividing the actual differences by the average values and multiplying by 100. The dark blue bars represent the mean absolute of the relative differences and the yellow bars show the standard deviation of them. The maximum relative differences were 4.9, 9.1, 9.2 and $6.5 \%$ for SDNN, rMSSD, SD1 and SD2, respectively. The A-S lead provided the smallest relative deviation among other leads for all the HRV parameters.

\section{B. Signal Quality}

To assist the lead selection for HRV analysis, the amplitude of all the R-peaks is reported as a measure of signal quality and signal-to-noise and artifact ratio. The amplitudes of the R-peaks were measured after removing the baseline wandering. Fig. 6 shows the distributions of the R-peak amplitudes in all the leads. It can be seen that A-S lead provides the largest R-peaks. Among the limb leads, lead II is the most appropriate lead to be used for this purpose.

\section{DISCUSSION AND CONCLUSIONS}

In this work, the deviation in heart rate and heart rate variability parameters with respect to several ECG leads was studied. Nine different leads from EASI and Mason-Likar lead sets were considered. Five subjects volunteered for this study and the data collection was performed in stationary 
condition while the subjects were laying down in supine posture. All the leads underwent the same signal processing method to characterize the corresponding RRI time series and HRV parameters derived from them. The values obtained from different leads were finally compared against average values obtained from all the leads.

In our experiment the maximum difference of an individual RRI value was approximately $5 \mathrm{~ms}$ from the average value of the corresponding $\mathrm{R}-\mathrm{R}$ interval in all the leads. While this difference is not particularly large, it still shows that the electrode locations i.e. the measurement lead has an effect on the obtained $\mathrm{R}-\mathrm{R}$ intervals. To our understanding this effect is due to changes in the orientation of the main electrical axis of the heart with respect to the measurement lead-field caused by breathing.

In the HRV parameters, the maximum deviation of a single value for a 5-minute RRI frame was $3.45 \mathrm{~ms}$ from the average. This occured in the SD2 parameter. The mean absolute relative deviation was less than $2 \%$ for all the HRV parameters. However, there were a few occasions where the HRV parameters showed significantly larger relative deviviation for a single 5-minute frame. The largest individual deviations, 9.1 and $9.2 \%$ were seen in rMSSD and SD1 parameters, respectively. As a general advice it should therefore be mentioned that relying on a single HRV parameter value, even if calculated from a commonly used 5minute RRI segment should be avoided when accurate results are desired. While the demonstrated uncertainty, e.g. max. 3 ms for rMSSD, is small compared to the average value of this parameter and its standard deviation, e.g. $40 \mathrm{~ms}$ (23-72 ms) for young adults as in [17](originally reported as logarithmic rMSSD), the relative error is increased in case the value of the HRV parameter is decreased such as in cases of elderly or during mental or physical stress.

The evaluation of the signal quality between different leads showed that A-S lead provides the most powerful R-peaks. This was expected due to the fact that this lead is the most aligned with the heart electrical axis during the contraction of ventricles. Additionally, this lead has an adequate inter-electrode distance, which is long enough that a large potential difference is sensed. It is noteworthy that this lead provided significantly larger R-peak amplitude that the lead II, which is often used in HRV measurements. Furthermore, it has previously been shown that this lead provides the most promising respiration cycle among other EASI leads using electrical impedance pneumography [16]. Therefore, this single lead can be used in a combinatory measurement of heart rate and respiration cycle.

Although the heart rate and heart rate variability parameters are usually assessed using the lead II of the 12-lead ECG, the lack of a standard lead for HRV analysis was one of the challenges in this study. For this reason, all the parameters have been compared against the mean, while the average itself might be different from the optimal values. It should also be noted that the definition of optimal may depend on the intended usage of the parameters (e.g. what phenomena are studied with them). Therefore, the results might be biased depending on the context.

In future studies, we will investigate other situations where HR and HRV are of interest e.g. for stress and fitness level assessment. A more thorough HRV analysis will be considered and different age groups and health conditions will be evaluated.

\section{REFERENCES}

[1] M. Buchheit, C. Gindre, Cardiac parasympathetic regulation: respective associations with cardiorespiratory fitness and training load, American Journal of Physiology-Heart and Circulatory Physiology, vol. 291, no. 1, 2006, H451-H458.

[2] R. E. De Meersman, Heart rate variability and aerobic fitness, American heart journal, vol. 125, no. 3, 1993, 726-731.

[3] J. A. Healey, R. W. Picard, Detecting stress during real-world driving tasks using physiological sensors, IEEE Transactions on intelligent transportation systems, vol. 6, no. 2, 2005, 156-166.

[4] A. L. Hansen, B. H. Johnsen, J. F. Thayer, Vagal influence on working memory and attention, International Journal of Psychophysiology, vol. 48 , no. 3, 2003, 263-274.

[5] V. Jeyhani, S. Mahdiani, M. Peltokangas, A. Vehkaoja, Comparison of HRV parameters derived from photoplethysmography and electrocardiography signals, Engineering in Medicine and Biology Society (EMBC), 2015 37th Annual International Conference of the IEEE, 2015, 5952-5955.

[6] A. Vehkaoja, S. Rajala, P. Kumpulainen, J. Lekkala, Correlation approach for the detection of the heartbeat intervals using force sensors placed under the bed posts, Journal of medical engineering \& technology, vol. 37, no 5, 2013, 327-333.

[7] S. Mahdiani, V. Jeyhani, M. Peltokangas, A. Vehkaoja, Is $50 \mathrm{~Hz}$ high enough ECG sampling frequency for accurate HRV analysis?, Engineering in Medicine and Biology Society (EMBC), 2015 37th Annual International Conference of the IEEE, 2015, 5948-5951.

[8] M. Puurtinen, J. Viik, J. Hyttinen, Best electrode locations for a small bipolar ECG device: Signal strength analysis of clinical data, Annals of biomedical engineering, vol. 37, no. 2, 2009, 331-336.

[9] H. W. Noh, Y. Jang, I. B. Lee, Y. Song, J W. Jeong, S. Lee, A preliminary study of the effect of electrode placement in order to define a suitable location for two electrodes and obtain sufficiently reliable ECG signals when monitoring with wireless system, Engineering in Medicine and Biology Society (EMBC), 2012 Annual International Conference of the IEEE, 2012, 2124-2127.

[10] V. Jeyhani, A Wireless Device for Ambulatory Cardiac and Respiratory Monitoring - Design Considerations and Essential Performance, Tampere University of Technology, Oct. 2017. URN.fi/URN:NBN:fi:tty201709251955

[11] G. E. Dower, A. Yakush, S. B. Nazzal, R. V. Jutzy, and C. E. Ruiz, Deriving the 12-lead electrocardiogram from four (EASI) electrodes, in Journal of electrocardiology, vol. 21, 1988, S182-S187.

[12] R. E. Mason, I. Likar, A new system of multiple-lead exercise electrocardiography, in American Heart Journal, 1966, vol. 71, no. 2.

[13] L. Keselbrener, M. Keselbrener S. Akselrod, Nonlinear high pass filter for R-wave detection in ECG signal, Medical engineering \& physics, vol. 19, no. 5, 1997, 481-484.

[14] G. Zhengzhong, K. Fanxue, Z. Xu, Accurate and rapid QRS detection for intelligent ECG monitor, Measuring Technology and Mechatronics Automation (ICMTMA), 2011 Third International Conference on, vol. 1, 2011, 298-301.

[15] Electrophysiology, Task Force of the European Society of Cardiology the North American Society of Pacing, Heart Rate Variability Standards of Measurement, Physiological Interpretation, and Clinical Use, American Heart Association, Inc., vol. 93, no. 5, 1996, 1043-1065.

[16] V. Jeyhani, T. Vuorinen, K. Noponen, M. Mäntysalo, A. Vehkaoja, Optimal short distance electrode locations for impedance pneumography measurement from the frontal thoracic area, XIV Mediterranean Conference on Medical and Biological Engineering and Computing 2016, 2016, 1144-1149.

[17] T. Koskinen, M. Kähönen, A. Jula, T. Laitinen, L. KeltikangasJärvinen, J. Viikari, I. Välimäki, O. Raitakari, Short-term heart rate variability in healthy young adults: the Cardiovascular Risk in Young Finns Study. Autonomic Neuroscience, vol. 145, no. 1-2, 2009, 81-88. 